\title{
Atmospheric effects on extensive air showers observed with the surface detector of the Pierre Auger observatory
}

The Pierre Auger Collaboration, J. Abraham ${ }^{g}$, P. Abreu ${ }^{\text {bj }}$, M. Aglietta ${ }^{\text {au }}$, C. Aguirre ${ }^{\text {k }}$, E.J. Ahn ${ }^{\text {by }}$, D. Allard ${ }^{\mathrm{ab}, \mathrm{bb}}$, I. Allekotte ${ }^{\mathrm{a}}$, J. Allen ${ }^{\mathrm{cb}}$, P. Allison ${ }^{\mathrm{cd}}$, J. Alvarez-Muñiz ${ }^{\mathrm{bq}}, \mathrm{M}$. Ambrosio ${ }^{\text {ar }}$, L. Anchordoqui ${ }^{\mathrm{cn}}$, S. Andringa ${ }^{\text {bj }}$, A. Anzalone ${ }^{\mathrm{av}}$, C. Aramo ${ }^{\mathrm{ar}}$, E. Arganda ${ }^{\mathrm{bn}}, \mathrm{S}$. Argirò ${ }^{\mathrm{au}}$, K. Arisaka ${ }^{\mathrm{cg}}, \mathrm{F}$. Arneodo ${ }^{\mathrm{ax}}$, F. Arqueros ${ }^{\text {bn }}$, T. Asch ${ }^{\text {ai }}$, H. Asorey ${ }^{a}$, P. Assis ${ }^{\text {bj }}$, J. Aublin ${ }^{\text {ad }}$, M. Ave ${ }^{\text {ch }}$, G. Avila ${ }^{i}$, T. Bäcker ${ }^{a m}$, D. Badagnani ${ }^{\mathrm{e}}$, K.B. Barber ${ }^{\mathrm{j}}$, A.F. Barbosa ${ }^{\mathrm{m}}$, S.L.C. Barroso ${ }^{\mathrm{s}}$, B. Baughman ${ }^{\mathrm{cd}}$, P. Bauleo ${ }^{\text {bw }}$, J.J. Beatty ${ }^{\mathrm{cd}}$, T. Beau ${ }^{\text {ab }}$, B.R. Becker ${ }^{\text {ck }}$, K.H. Becker ${ }^{\text {ag }}$, A. Bellétoile ${ }^{\text {ae }}$, J.A. Bellido ${ }^{\mathrm{j}, \mathrm{cg}}, \mathrm{S}$. BenZvi ${ }^{\mathrm{cm}}$, C. Berat $^{\text {ae }}$, P. Bernardini ${ }^{\text {aq }}$, X. Bertou ${ }^{\text {a }}$, P.L. Biermann ${ }^{\text {aj }}$, P. Billoir ${ }^{\text {ad }}$, O. Blanch-Bigas ${ }^{\text {ad }}$, F. Blanco $^{\text {bn }}$, C. Bleve ${ }^{\text {aq }, *}$, H. Blümer ${ }^{\text {al,ah }}$, M. Boháčová ch,y, C. Bonifazi ${ }^{\text {ad }}$, R. Bonino $^{\text {aw }}$, N. Borodai ${ }^{\text {bh }}$, J. Brack ${ }^{\text {bw }}$, P. Brogueira ${ }^{\text {bj }}$, W.C. Brown ${ }^{\text {bx }}$, R. Bruijn ${ }^{\text {bs }}$, P. Buchholz ${ }^{a m}$, A. Bueno ${ }^{\text {bp }}$, R.E. Burton ${ }^{\text {bu }}$, N.G. Busca ${ }^{\text {ab }}$, K.S. Caballero-Mora ${ }^{a l}$, L. Caramete $^{\mathrm{aj}}$, R. Caruso $^{\text {at }}$, W. Carvalho ${ }^{\mathrm{p}}, \mathrm{A}$. Castellina ${ }^{\mathrm{aw}}$, O. Catalano $^{\mathrm{av}}$, L. Cazon $^{\text {ai }}$, R. Cester $^{\mathrm{au}}$, J. Chauvin ${ }^{\mathrm{ae}}$, A. Chiavassa ${ }^{\mathrm{aw}}$, J.A. Chinellato ${ }^{\mathrm{q}}$, A. Chou ${ }^{\text {by,cb }}{ }^{\mathrm{w}}$ J. Chudoba ${ }^{\mathrm{y}}$, J. Chye ${ }^{\text {ca }}$, R.W. Clay ${ }^{\mathrm{j}}$, E. Colombo ${ }^{\text {b }}$, R. Conceição ${ }^{\text {bj }}$, B. Connolly ${ }^{c l}$, F. Contreras ${ }^{\text {h }}$, J. Coppens ${ }^{\text {bd,bf }}$, A. Cordier ${ }^{\text {ac }}$, U. Cotti $^{\text {bb }}$, S. Coutu ${ }^{\text {ce }}$, C.E. Covault ${ }^{\text {bu }}$, A. Creusot ${ }^{\text {bl }}$, A. Criss ${ }^{\text {ce }}$, J. Cronin ${ }^{\text {ch }}$, A. Curutiu ${ }^{\text {aj }}$, S. Dagoret-Campagne ${ }^{\text {ac }}$, R. Dallier $^{\text {af }}$, K. Daumiller ${ }^{\text {ah }}$, B.R. Dawson ${ }^{\mathrm{j}}$, R.M. de Almeida ${ }^{\mathrm{q}}$, M. De Domenico ${ }^{\text {at }}$, C. De Donato ${ }^{\text {ap }}$, S.J. de Jong ${ }^{\text {bd }}$, G. De La Vega ${ }^{\mathrm{g}}$, W.J.M. de Mello Junior ${ }^{\mathrm{q}}$, J.R.T. de Mello Neto ${ }^{\mathrm{v}}$, I. De Mitri ${ }^{\mathrm{aq}}$, V. de Souza ${ }^{\mathrm{o}}$, K.D. de Vries ${ }^{\text {be }}$, G. Decerprit $^{\mathrm{ab}}$, L. del Peral ${ }^{\text {bo }}$, O. Deligny ${ }^{\mathrm{aa}}$, A. Della Selva ${ }^{\mathrm{ar}}$, C. Delle Fratte $^{\mathrm{as}}$, H. Dembinski $^{\mathrm{ak}}$, C. Di Giulio ${ }^{\text {as }}$, J.C. Diaz ${ }^{\text {ca }}$, P.N. Diep ${ }^{\text {co }}$, C. Dobrigkeit ${ }^{\mathrm{q}}$, J.C. D’Olivo ${ }^{\text {bc }}$, P.N. Dong ${ }^{c o}$, D. Dornic ${ }^{\text {aa }}$, A. Dorofeev ${ }^{\text {bz }}$, J.C. dos Anjos ${ }^{\mathrm{m}}$, M.T. Dova ${ }^{\mathrm{e}}$, D. D’Urso ${ }^{\mathrm{ar}}$, I. Dutan ${ }^{\text {aj }}$, M.A. DuVernois ${ }^{\mathrm{ci}}$, R. Engel ${ }^{\text {ah }}$, M. Erdmann ${ }^{\text {ak }}$, C.O. Escobar ${ }^{\mathrm{q}}$, A. Etchegoyen ${ }^{\text {b }}$, P. Facal San Luis ${ }^{\text {ch,bq }}$, H. Falcke ${ }^{\text {bd,bg }}$, G. Farrar $^{\text {cb }}$,


S. Fliescher ${ }^{\text {ak }}$, C.E. Fracchiolla ${ }^{n}$, E.D. Fraenkel ${ }^{\text {be }}$, W. Fulgione ${ }^{\text {aw }}$, R.F. Gamarra ${ }^{\text {b }}$, S. Gambetta ${ }^{\text {an }}$, B. García ${ }^{g}$, D. García Gámez ${ }^{\text {bp }}$, D. Garcia-Pinto ${ }^{\text {bn }}$, X. Garrido ${ }^{\text {ah,ac }}{ }^{\text {G. G. Gelmini }}{ }^{\text {cg }}$, H. Gemmeke ${ }^{\text {ai }}$, P.L. Ghia ${ }^{\text {aa,aw }}$, U. Giaccari ${ }^{\text {aq }}$, M. Giller ${ }^{\text {bi }}$, H. Glass ${ }^{\text {by }}$, L.M. Goggin ${ }^{\text {cn }}$, M.S. Gold ${ }^{\text {ck }}$, G. Golup ${ }^{\text {a }}$, F. Gomez Albarracin ${ }^{\mathrm{e}}$, M. Gómez Berisso ${ }^{\mathrm{a}}$, P. Gonçalves ${ }^{\mathrm{bj}}$, M. Gonçalves do Amaral w , D. Gonzalez ${ }^{\text {al }}$, J.G. Gonzalez ${ }^{\text {bp,by }}{ }^{\text {, D. Góra }}{ }^{\text {al,bh }}$, A. Gorgi aw , P. Gouffon ${ }^{\text {p }}$, E. Grashorn ${ }^{\text {cd }}$, S. Grebe ${ }^{\text {bd }}$, M. Grigat ${ }^{\text {ak }}$, A.F. Grillo ${ }^{\text {ax }}$, Y. Guardincerri $^{\mathrm{d}}$, F. Guarino ar , G.P. Guedes ${ }^{r}$, J. Gutiérrez ${ }^{\text {bo }}$, J.D. Hague ${ }^{c k}$, V. Halenka ${ }^{z}$, P. Hansen ${ }^{\text {e }}$, D. Harari ${ }^{\text {a }}$, S. Harmsma be,bf , J.L. Harton ${ }^{\text {bw }}$, A. Haungs ${ }^{\text {ah }}$, M.D. Healy ${ }^{\text {cg }}$, T. Hebbeker ${ }^{\text {ak }}$,

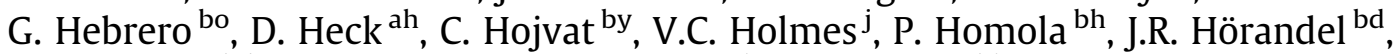
A. Horneffer ${ }^{\text {bd }}$, M. Hrabovský ${ }^{z, y}$, T. Huege $^{\text {ah }}$, M. Hussain ${ }^{\text {bl }}$, M. Iarlori ${ }^{\text {ao }}$, A. Insolia $^{\text {at }}$, F. Ionita ${ }^{\text {ch }}$, A. Italiano ${ }^{\text {at }}$, S. Jiraskova ${ }^{\text {bd }}$, M. Kaducak ${ }^{\text {by }}$, K.H. Kampert ${ }^{\text {ag }}$, T. Karova ${ }^{\mathrm{y}}$, P. Kasper ${ }^{\text {by }}$, B. Kégl ${ }^{\text {ac }}$, B. Keilhauer ${ }^{\text {ah }}$, E. Kemp ${ }^{\text {q }}$, R.M. Kieckhafer ${ }^{\text {cc }}$, H.O. Klages ${ }^{\text {ah }}$, M. Kleifges ${ }^{\text {ai }}$, J. Kleinfeller ${ }^{\text {ah }}$, R. Knapik ${ }^{\text {bw }}$, J. Knapp ${ }^{\text {bs }}$, D.-H. Koang ${ }^{\text {ae }}$, A. Krieger ${ }^{\text {b }}$, O. Krömer ${ }^{\text {ai }}$, D. Kruppke-Hansen ${ }^{\text {ag }}$, D. Kuempel ${ }^{\text {ag }}$, N. Kunka ${ }^{\text {ai }}$, A. Kusenko ${ }^{\text {cg, }}$, G. La Rosa ${ }^{\text {av }}$, C. Lachaud ${ }^{\text {ab }}$, B.L. Lago v, P. Lautridou ${ }^{\text {af }}$, M.S.A.B. Leão ${ }^{u}$, D. Lebrun ${ }^{\text {ae }}$, P. Lebrun ${ }^{\text {by }}$, J. Lee ${ }^{\text {cg }}$, M.A. Leigui de Oliveira ${ }^{\mathrm{u}}$, A. Lemiere $^{\text {aa }}$, A. Letessier-Selvon ${ }^{\text {ad }}$, M. Leuthold ${ }^{\text {ak }}$, I. Lhenry-Yvon ${ }^{\text {aa }}$, R. López ${ }^{\text {ay }}$, A. Lopez Agüera ${ }^{\text {bq }}$, K. Louedec $^{\text {ac }}$, J. Lozano Bahilo $^{\text {bp }}$, A. Lucero ${ }^{\text {aw }}$, R. Luna García ${ }^{\text {ac }}$, H. Lyberis ${ }^{\text {aa }}$, M.C. Maccarone ${ }^{\text {av }}$, C. Macolino $^{\text {ao }}$, S. Maldera ${ }^{\text {aw }}$, D. Mandat ${ }^{y}$, P. Mantsch ${ }^{\text {by }}$, A.G. Mariazzi ${ }^{\mathrm{e}}$, I.C. Maris ${ }^{\text {al }}$, 
H.R. Marquez Falcon ${ }^{\text {bb }}$, D. Martello aq, J. Martínez ${ }^{\text {ba }}$, O. Martínez Bravo ${ }^{\text {ay }}$, H.J. Mathes ${ }^{\text {ah }}$, J. Matthews ${ }^{\text {bz,cf }}$, J.A.J. Matthews ${ }^{\text {ck }}$, G. Matthiae ${ }^{\text {as }}$, D. Maurizio ${ }^{\text {au }}$, P.O. Mazur ${ }^{\text {by }}$, M. McEwen ${ }^{\text {bo }}$, R.R. McNeil ${ }^{\text {bz }}$, G. Medina-Tanco ${ }^{\text {bc }}$, M. Melissas ${ }^{\text {al }}$, D. Melo ${ }^{\text {au }}$, E. Menichetti $^{\text {au }}$, A. Menshikov ${ }^{\text {ai }}$, R. Meyhandan ${ }^{\text {be }}$, M.I. Micheletti ${ }^{\text {b }}$, G. Miele ${ }^{\text {ar }}$, W. Miller ${ }^{\mathrm{ck}}$, L. Miramonti ${ }^{\text {ap }}$, S. Mollerach ${ }^{\mathrm{a}}$, M. Monasor ${ }^{\mathrm{bn}}{ }^{\text {, D. Monnier Ragaigne }}{ }^{\mathrm{ac}}$, F. Montanet ${ }^{\mathrm{ae}}$, B. Morales ${ }^{\mathrm{bc}}$, C. Morello ${ }^{\mathrm{aw}}$, J.C. Moreno $^{\mathrm{e}}$, C. Morris $^{\mathrm{cb}}$, M. Mostafá bw C.A. Moura ${ }^{\text {ar }}$, S. Mueller ${ }^{\text {ah }}$, M.A. Muller ${ }^{\mathrm{q}}$, R. Mussa ${ }^{\text {au }}$, G. Navarra ${ }^{\text {aw }}$,

J.L. Navarro ${ }^{\text {bp }}$, S. Navas ${ }^{\text {bp }}$, P. Necesal ${ }^{\text {y, }}$ L. Nellen ${ }^{\text {be }}$, C. Newman-Holmes ${ }^{\text {by }}$, D. Newton ${ }^{\text {bs }}$, P.T. Nhung ${ }^{\text {co }}$, N. Nierstenhoefer ${ }^{\text {ag }}$, D. Nitz ${ }^{\text {ca }}$, D. Nosek ${ }^{\mathrm{x}}$, L. Nožka ${ }^{\mathrm{y}}$, M. Nyklicek ${ }^{\mathrm{y}}$, J. Oehlschläger $^{\text {ah }}$, A. Olinto ${ }^{\mathrm{ch}}$, P. Oliva ${ }^{\mathrm{ag}}$, V.M. Olmos-Gilbaja ${ }^{\text {bq }}$, M. Ortiz ${ }^{\text {bn }}$, F. Ortolani ${ }^{\text {as }}$, N. Pacheco ${ }^{\text {bo }}$, D. Pakk Selmi-Dei ${ }^{\text {, }}$, M. Palatka ${ }^{\mathrm{y}}$, J. Pallotta ${ }^{\mathrm{c}}$, G. Parente $^{\mathrm{bq}}$, E. Parizot $^{\mathrm{ab}}$, S. Parlati ${ }^{\mathrm{ax}}$, S. Pastor ${ }^{\text {bm }}$, M. Patel ${ }^{\text {bs }}$, T. Paul ${ }^{\text {cc }}$, V. Pavlidou ${ }^{\text {ch,1 }}$, K. Payet ${ }^{\text {ae }}$, M. Pech ${ }^{\mathrm{y}}$, J. Pȩkala ${ }^{\text {bh }}$, R. Pelayo ${ }^{\text {bb }}$, I.M. Pepe ${ }^{t}$, L. Perrone ${ }^{a q}$, R. Pesce $^{\text {an }}$, E. Petermann $^{c j}$, S. Petrera ${ }^{\text {ao }}$, P. Petrinca as, A. Petrolini an ${ }^{\text {an }}$ Y. Petrov ${ }^{\text {bw }}$, J. Petrovic ${ }^{\text {bg }}$, C. Pfendner ${ }^{\mathrm{cm}}$, R. Piegaia $^{\mathrm{d}}$, T. Pierog ${ }^{\text {ah }}$, M. Pimenta ${ }^{\text {bj }}$, T. Pinto $^{\text {bm }}$, V. Pirronello ${ }^{\text {at }}$, O. Pisanti $^{\text {ar }}$, M. Platino ${ }^{\mathrm{b}}$, J. Pochon ${ }^{\mathrm{a}}$, V.H. Ponce ${ }^{\mathrm{a}}$, M. Pontz ${ }^{\mathrm{am}}$, P. Privitera ${ }^{\mathrm{ch}}$, M. Prouza ${ }^{\mathrm{y}}$, E.J. Quel ${ }^{\text {c }}$, J. Rautenberg ${ }^{\text {ag }}$, O. Ravel ${ }^{\text {af }}$, D. Ravignani ${ }^{\text {b }}$, A. Redondo ${ }^{\text {bo }}$, S. Reucroft ${ }^{c c}$, B. Revenu ${ }^{\text {af }}$, F.A.S. Rezende ${ }^{\mathrm{m}}$, J. Ridky ${ }^{\mathrm{y}}$, S. Riggi ${ }^{\text {at }}$, M. Risse ${ }^{\mathrm{ag}}$, C. Rivière $^{\mathrm{ae}}$, V. Rizi ${ }^{\text {ao }}$, C. Robledo ay, G. Rodriguez ${ }^{\text {as }}$, J. Rodriguez Martino ${ }^{\text {at }}$, J. Rodriguez Rojo ${ }^{\mathrm{h}}$, I. Rodriguez-Cabo $^{\text {bs }}$, M.D. Rodríguez-Frías ${ }^{\text {bo }}$, G. Ros $^{\text {bn,bo }}{ }^{\text {, J. Rosado }}{ }^{\text {bn }}$, T. Rossler ${ }^{z}$, M. Roth ${ }^{\text {ah }}$, A. Rouillé-d'Orfeuil ${ }^{\text {ab }}$, E. Roulet ${ }^{a}$, A.C. Rovero ${ }^{\mathrm{f}}$, F. Salamida ${ }^{\text {ao }}$, H. Salazar ${ }^{\text {ay,2 }}$, G. Salina ${ }^{\text {as }}$, F. Sánchez ${ }^{\text {bc }}$, M. Santander ${ }^{\text {h }}$, C.E. Santo ${ }^{\text {bj }}$, E.M. Santos ${ }^{\text {v }}$, F. Sarazin ${ }^{\text {bv }}$, S. Sarkar ${ }^{\text {br }}$, R. Sato ${ }^{\text {h }}$, N. Scharf ${ }^{\text {ak }}$, V. Scherini ${ }^{\text {ag }}$, H. Schieler ${ }^{\text {ah }}$, P. Schiffer ${ }^{\text {ak }}$, A. Schmidt ${ }^{\text {ai }}$, F. Schmidt ${ }^{\text {ch }}$, T. Schmidt ${ }^{\text {av }}$, O. Scholten ${ }^{\text {be }}$, H. Schoorlemmer ${ }^{\text {bd }}$, J. Schovancova ${ }^{\mathrm{y}}$, P. Schovánek ${ }^{\mathrm{y}}$, F. Schroeder ${ }^{\text {ah }}$, S. Schulte ${ }^{\mathrm{ak}}$, F. Schüssler ${ }^{\text {ah }}$, D. Schuster ${ }^{\text {bv }}$, S.J. Sciutto ${ }^{\text {e, M. Scuderi }}{ }^{\text {at }}$, A. Segreto ${ }^{\text {av }}$,

D. Semikoz ${ }^{\text {ab }}$, M. Settimo ${ }^{\text {aq }}$, R.C. Shellard ${ }^{\mathrm{m}, \mathrm{n}}$, I. Sidelnik ${ }^{\mathrm{b}}$, B.B. Siffert ${ }^{\mathrm{v}}$,

A. Smiałkowski ${ }^{\text {bi }}$, R. Šmída ${ }^{y}$, B.E. Smith ${ }^{\text {bk }}$, G.R. Snow ${ }^{c j}$, P. Sommers ${ }^{c e}$, J. Sorokin ${ }^{j}$, H. Spinka ${ }^{\text {bt,by }}$, R. Squartini ${ }^{\text {h, E. Strazzeri }}{ }^{\text {ac }}$, A. Stutz $^{\text {ae }}$, F. Suarez ${ }^{\text {b }}$, T. Suomijärvi ${ }^{\text {aa }}$, A.D. Supanitsky ${ }^{\text {bc }}$, M.S. Sutherland ${ }^{\text {cd }}$, J. Swain ${ }^{c c}$, Z. Szadkowski ${ }^{\text {bi }}$, A. Tamashiro ${ }^{\text {f }}$ ' A. Tamburro $^{\text {al }}$, T. Tarutina ${ }^{\mathrm{e}}$, O. Taşcău $^{\text {ag }}$, R. Tcaciuc ${ }^{\text {am }}$, D. Tcherniakhovski ${ }^{\text {ai }}$, N.T. Thao ${ }^{\text {co }}$, D. Thomas ${ }^{\text {bw }}$, R. Ticona ${ }^{1}$, J. Tiffenberg ${ }^{d}$, C. Timmermans ${ }^{\text {bf,bd, }}{ }^{\text {, }}$ W. Tkaczyk ${ }^{\text {bi }}$, C.J. Todero Peixoto ${ }^{\text {u }}$, B. Tomé ${ }^{\text {bj }}$, A. Tonachini ${ }^{\text {au }}$, I. Torres $^{\text {ay }}$, P. Travnicek ${ }^{\mathrm{y}}$, D.B. Tridapalli ${ }^{\mathrm{p}}$, G. Tristram ${ }^{\text {ab }}$, E. Trovato ${ }^{\text {at }}$, V. Tuci ${ }^{\text {as }}$, M. Tueros ${ }^{\mathrm{e}}$, R. Ulrich ${ }^{\text {ah }}$, M. Unger $^{\text {ah }}$, M. Urban ${ }^{\text {aa }}$, J.F. Valdés Galicia ${ }^{\text {bc }}$, I. Valiño $^{\text {ah }}$, L. Valore ${ }^{\text {ar }}$, A.M. van den Berg ${ }^{\text {be }}$, J.R. Vázquez ${ }^{\text {bn }}$, R.A. Vázquez ${ }^{\text {bq }}$, D. Veberič ${ }^{\text {bl,bm }}{ }^{\text {, A. Velarde }}{ }^{\text {, }}$, T. Venters ${ }^{\text {ch }}$, V. Verzi ${ }^{\text {as }}$, M. Videla ${ }^{g}$, L. Villaseñor ${ }^{\text {bb }}$, S. Vorobiov ${ }^{\text {bl }}$, L. Voyvodic ${ }^{\text {by, }}{ }^{3}$, H. Wahlberg ${ }^{\mathrm{e}}$, P. Wahrlich ${ }^{j}$, O. Wainberg ${ }^{b}$, D. Warner ${ }^{\text {bw }}$, A.A. Watson ${ }^{\text {bs }}$, S. Westerhoff ${ }^{c m}$, B.J. Whelan ${ }^{j}$, G. Wieczorek ${ }^{\text {bi }}$, L. Wiencke $^{\text {bw }}$, B. Wilczyńska ${ }^{\text {bh }}$, H. Wilczyński ${ }^{\text {bh }}$, C. Wileman ${ }^{\text {bs }}$, M.G. Winnick ${ }^{\mathrm{j}}, \mathrm{H}$ Wu $^{\text {ac }}$, B. Wundheiler $^{\mathrm{b}}$, T. Yamamoto ${ }^{\text {ch,4 }}$, P. Younk ${ }^{\text {bw }}$, G. Yuan ${ }^{\text {bz }}$, E. Zas ${ }^{\text {bq }}$, D. Zavrtanik ${ }^{\text {bl,bm }}$, M. Zavrtanik ${ }^{\text {bm,bl }}$, I. Zaw ${ }^{\text {cb }}$, A. Zepeda ${ }^{a z, 2}$, M. Ziolkowski ${ }^{\text {am }}$

\footnotetext{
${ }^{a}$ Centro Atómico Bariloche and Instituto Balseiro (CNEA-UNCuyo-CONICET), San Carlos de Bariloche, Argentina



${ }^{\mathrm{c}}$ Centro de Investigaciones en Láseres y Aplicaciones, CITEFA and CONICET, Argentina

${ }^{\mathrm{d}}$ Departamento de Física, FCEyN, Universidad de Buenos Aires y CONICET, Argentina

e IFLP, Universidad Nacional de La Plata and CONICET, La Plata, Argentina

f Instituto de Astronomía y Física del Espacio (CONICET), Buenos Aires, Argentina

${ }^{g}$ Observatorio Meteorologico Parque Gral. San Martin (UTN- FRM/CONICET/CNEA), Mendoza, Argentina

${ }^{\mathrm{h}}$ Pierre Auger Southern Observatory, Malargüe, Argentina

i Pierre Auger Southern Observatory and Comisión Nacional de Energía Atómica, Malargüe, Argentina

${ }^{\mathrm{j}}$ University of Adelaide, Adelaide, S.A., Australia

${ }^{\mathrm{k}}$ Universidad Catolica de Bolivia, La Paz, Bolivia

${ }^{1}$ Universidad Mayor de San Andrés, Bolivia

${ }^{\mathrm{m}}$ Centro Brasileiro de Pesquisas Fisicas, Rio de Janeiro, RJ, Brazil

${ }^{\mathrm{n}}$ Pontifícia Universidade Católica, Rio de Janeiro, RJ, Brazil
} 
${ }^{\circ}$ Universidade de São Paulo, Instituto de Física, São Carlos, SP, Brazil

${ }^{\mathrm{P}}$ Universidade de São Paulo, Instituto de Física, São Paulo, SP, Brazi

${ }^{\mathrm{q}}$ Universidade Estadual de Campinas, IFGW, Campinas, SP, Brazil

${ }^{\mathrm{r}}$ Universidade Estadual de Feira de Santana, Brazil

${ }^{\mathrm{s}}$ Universidade Estadual do Sudoeste da Bahia, Vitoria da Conquista, BA, Brazil

${ }^{\mathrm{t}}$ Universidade Federal da Bahia, Salvador, BA, Brazil

universidade Federal do ABC, Santo André, SP, Brazil

${ }^{\mathrm{v}}$ Universidade Federal do Rio de Janeiro, Instituto de Física, Rio de Janeiro, RJ, Brazil

${ }^{\mathrm{w}}$ Universidade Federal Fluminense, Instituto de Fisica, Niterói, RJ, Brazil

${ }^{x}$ Charles University, Faculty of Mathematics and Physics, Institute of Particle and Nuclear Physics, Prague, Czech Republic

${ }^{\mathrm{y}}$ Institute of Physics of the Academy of Sciences of the Czech Republic, Prague, Czech Republic

${ }^{z}$ Palacký University, Olomouc, Czech Republic

aa Institut de Physique Nucléaire d'Orsay (IPNO), Université Paris 11, CNRS-IN2P3, Orsay, France

${ }^{\mathrm{ab}}$ Laboratoire AstroParticule et Cosmologie (APC), Université Paris 7, CNRS-IN2P3, Paris, France

ac Laboratoire de l'Accélérateur Linéaire (LAL), Université Paris 11, CNRS-IN2P3, Orsay, France

${ }^{\mathrm{ad}}$ Laboratoire de Physique Nucléaire et de Hautes Energies (LPNHE), Universités Paris 6 et Paris 7, CNRS-IN2P3, Paris Cedex 05, France

${ }^{\text {ae }}$ Laboratoire de Physique Subatomique et de Cosmologie (LPSC), Université Joseph Fourier, INPG, CNRS-IN2P3, Grenoble, France

af SUBATECH, CNRS-IN2P3, Nantes, France

ag Bergische Universität Wuppertal, Wuppertal, Germany

${ }^{\text {ah }}$ Forschungszentrum Karlsruhe, Institut für Kernphysik, Karlsruhe, Germany

ai Forschungszentrum Karlsruhe, Institut für Prozessdatenverarbeitung und Elektronik, Germany

${ }^{\mathrm{aj}}$ Max-Planck-Institut für Radioastronomie, Bonn, Germany

${ }^{\mathrm{ak}}$ RWTH Aachen University, III. Physikalisches Institut A, Aachen, Germany

${ }^{a 1}$ Universität Karlsruhe (TH), Institut für Experimentelle Kernphysik (IEKP), Karlsruhe, Germany

${ }^{\text {am }}$ Universität Siegen, Siegen, Germany

an Dipartimento di Fisica dell'Università and INFN, Genova, Italy

${ }^{\text {ao } U n i v e r s i t a ̀ ~ d e l l ' A q u i l a ~ a n d ~ I N F N, ~ L ' A q u i l a, ~ I t a l y ~}$

ap Università di Milano and Sezione INFN, Milan, Italy

${ }^{\mathrm{aq}}$ Dipartimento di Fisica dell'Università del Salento and Sezione INFN, Lecce, Italy

ar Università di Napoli "Federico II" and Sezione INFN, Napoli, Italy

as Università di Roma II "Tor Vergata" and Sezione INFN, Roma, Italy

at Università di Catania and Sezione INFN, Catania, Italy

${ }^{\mathrm{au}}$ Università di Torino and Sezione INFN, Torino, Italy

av Istituto di Astrofisica Spaziale e Fisica Cosmica di Palermo (INAF), Palermo, Italy

${ }^{\mathrm{aw}}$ Istituto di Fisica dello Spazio Interplanetario (INAF), Università di Torino and Sezione INFN, Torino, Italy

ax INFN, Laboratori Nazionali del Gran Sasso, Assergi (L'Aquila), Italy

ay Benemérita Universidad Autónoma de Puebla, Puebla, Mexico

${ }^{\mathrm{az}}$ Centro de Investigación y de Estudios Avanzados del IPN (CINVESTAV), México, DF, Mexico

ba Instituto Politécnico Nacional, México, DF, Mexico

${ }^{\mathrm{bb}}$ Universidad Michoacana de San Nicolas de Hidalgo, Morelia, Michoacan, Mexico

${ }^{\mathrm{bc}}$ Universidad Nacional Autonoma de Mexico, Mexico, DF, Mexico

bd IMAPP, Radboud University, Nijmegen, The Netherlands

be Kernfysisch Versneller Instituut, University of Groningen, Groningen, The Netherlands

${ }^{\text {bf }}$ NIKHEF, Amsterdam, The Netherlands

bg ASTRON, Dwingeloo, The Netherlands

${ }^{b h}$ Institute of Nuclear Physics PAN, Krakow, Poland

bi University of Łódź, Łódz, Poland

${ }^{\text {bj }}$ LIP and Instituto Superior Técnico, Lisboa, Portugal

${ }^{\mathrm{bk}}$ J. Stefan Institute, Ljubljana, Slovenia

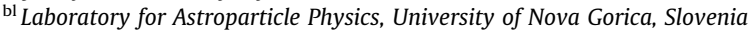

bm Instituto de Física Corpuscular, CSIC-Universitat de València, Valencia, Spain

bn Universidad Complutense de Madrid, Madrid, Spain

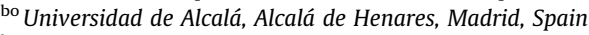

${ }^{\mathrm{bp}}$ Universidad de Granada \& C.A.F.P.E., Granada, Spain

${ }^{\mathrm{bq}}$ Universidad de Santiago de Compostela, Spain

${ }^{\text {br }}$ Rudolf Peierls Centre for Theoretical Physics, University of Oxford, Oxford, United Kingdom

${ }^{\text {bs }}$ School of Physics and Astronomy, University of Leeds, United Kingdom

${ }^{b t}$ Argonne National Laboratory, Argonne, IL, USA

bu Case Western Reserve University, Cleveland, $\mathrm{OH}$, USA

bv Colorado School of Mines, Golden, CO, USA

${ }^{\text {bw }}$ Colorado State University, Fort Collins, CO, USA

bx Colorado State University, Pueblo, CO, USA

by Fermilab, Batavia, IL, USA

${ }^{\mathrm{bz}}$ Louisiana State University, Baton Rouge, LA, USA

ca Michigan Technological University, Houghton, MI, USA

${ }^{\mathrm{cb}}$ New York University, New York, NY, USA

${ }^{\mathrm{cc}}$ Northeastern University, Boston, MA, USA

${ }^{\mathrm{cd}}$ Ohio State University, Columbus, OH, USA

ce Pennsylvania State University, University Park, PA, USA

${ }^{\mathrm{cf}}$ Southern University, Baton Rouge, LA, USA

${ }^{\mathrm{cg}}$ University of California, Los Angeles, CA, USA

${ }^{c h}$ University of Chicago, Enrico Fermi Institute, Chicago, IL, USA

${ }^{c i}$ University of Hawaii, Honolulu, HI, USA

${ }^{\mathrm{cj}}$ University of Nebraska, Lincoln, NE, USA

${ }^{\mathrm{ck}}$ University of New Mexico, Albuquerque, NM, USA

${ }^{\mathrm{cl}}$ University of Pennsylvania, Philadelphia, PA, USA

${ }^{\mathrm{cm}}$ University of Wisconsin, Madison, WI, USA 
cn University of Wisconsin, Milwaukee, WI, USA

${ }^{\mathrm{co}}$ Institute for Nuclear Science and Technology (INST), Hanoi, Viet Nam

\section{A R T I C L E I N F O}

\section{Article history:}

Received 28 May 2009

Received in revised form 22 June 2009

Accepted 22 June 2009

Available online 3 July 2009

\section{PACS:}

96.50.sd

96.50.sb

96.50.sf

Keywords:

Extensive air showers

UHECR

Atmosphere

Weather

\begin{abstract}
A B S T R A C T
Atmospheric parameters, such as pressure $(P)$, temperature $(T)$ and density $(\rho \propto P / T)$, affect the development of extensive air showers initiated by energetic cosmic rays. We have studied the impact of atmospheric variations on extensive air showers by means of the surface detector of the Pierre Auger Observatory. The rate of events shows a $\sim 10 \%$ seasonal modulation and $\sim 2 \%$ diurnal one. We find that the observed behaviour is explained by a model including the effects associated with the variations of $P$ and $\rho$. The former affects the longitudinal development of air showers while the latter influences the Molière radius and hence the lateral distribution of the shower particles. The model is validated with full simulations of extensive air showers using atmospheric profiles measured at the site of the Pierre Auger Observatory.
\end{abstract}

(c) 2009 Elsevier B.V. All rights reserved.

\section{Introduction}

High-energy cosmic rays (CRs) are measured by recording the extensive air showers (EAS) of secondary particles they produce in the atmosphere. As the atmosphere is the medium in which the shower evolves, its state affects the lateral and longitudinal development of the shower. Pressure $(P)$ and air density $(\rho)$ are the properties of the atmosphere that mostly affect the EAS. An increase (or decrease) of the ground $P$ corresponds to an increased (or decreased) amount of matter traversed by the shower particles; this affects the stage of the longitudinal development of the shower when it reaches the ground. A decrease (or increase) of $\rho$ increases (or decreases) the Molière radius and thus broadens (or narrows) the lateral extent of the EAS.

The properties of the primary $\mathrm{CR}$, e.g., energy, mass and arrival direction, have to be inferred from EAS, which can be sampled by an array of detectors at ground level. Therefore, the study and understanding of the effects of atmospheric variations on EAS in general, and on a specific detector in particular, is very important for the comprehension of the detector performances and for the correct interpretation of EAS measurements.

We have studied the atmospheric effects on EAS by means of the surface detector (SD) of the Pierre Auger Observatory, located in Malargüe, Argentina $\left(35.2^{\circ} \mathrm{S}, 69.5^{\circ} \mathrm{W}\right)$ at $1400 \mathrm{~m}$ a.s.l. [1]. The Pierre Auger Observatory is designed to study CRs from $\sim 10^{18} \mathrm{eV}$ up to the highest energies. The SD consists of 1600 water-Cherenkov detectors to detect the photons and the charged particles of the showers. It is laid out over $3000 \mathrm{~km}^{2}$ on a triangular grid of $1.5 \mathrm{~km}$ spacing [2] and is overlooked by four fluorescence detectors (FD) [3]. The SD trigger condition, based on a 3-station coincidence [4], makes the array fully efficient above about $3 \times 10^{18} \mathrm{eV}$. For each event, the signals in the stations are fitted to find the signal at $1000 \mathrm{~m}$ from the shower core, $S(1000)$, which is used to estimate the primary energy [5]. The atmosphere is continuously monitored by different meteorological stations located at the central part of the array and at each FD site. In addition, balloon-borne sensors are launched at regular intervals to measure the atmospheric temperature $T(h)$, pressure $P(h)$ and humidity $u(h)$ as a function of the altitude $h$ above the detector [6].

In Section 2, we develop a model of the expected atmospheric effects on $S(1000)$. The modulation is described by means of three coefficients that depend on the EAS zenith angle $(\theta)$. They are related to variations of $P$ and $\rho$, measured at ground level, on slower (daily-averaged) and faster (within a day) time scales. The dependence of $S(1000)$ on $P$ and $\rho$ implies a modulation of the counting rate of events. In Section 3, we study the behaviour of the recorded rate of events as a function of $P$ and $\rho$. On the base of the model defined previously, we derive the $P$ and $\rho$ coefficients. In Section 4 , we perform full simulations of EAS developing in various realistic atmospheres (based on measurements from balloon soundings above the site of the Pierre Auger Observatory) in order to compare, in Section 5, the results from data and simulations with the predictions of the model. We conclude in Section 6.

\section{Model of atmospheric effects for the surface detector of the Auger Observatory}

\subsection{Atmospheric effects on the measured signal}

The water-Cherenkov detectors are sensitive to both the electromagnetic component and the muonic component of the EAS, which are influenced to a different extent by atmospheric effects, namely by variations of $P$ and $\rho$. These in turn influence the signal measured in the detectors: for the Auger Observatory, we are in particular interested in the effects on the signal at $1000 \mathrm{~m}$ from the core, $S(1000)$.

The continuous measurement of atmospheric $P$ and $\rho$ is available only at ground level. We will show that the variation of $S(1000)$ can be fully described in terms of variation of air pressure and air density measured at the altitude of the Observatory site. If not otherwise stated, $P$ and $\rho$ refer to the values at ground level.

\footnotetext{
* Address: Dipartimento di Fisica dell'Università del Salento and Sezione INFN, Lecce, Italy. Tel.: +39 0832297465. 


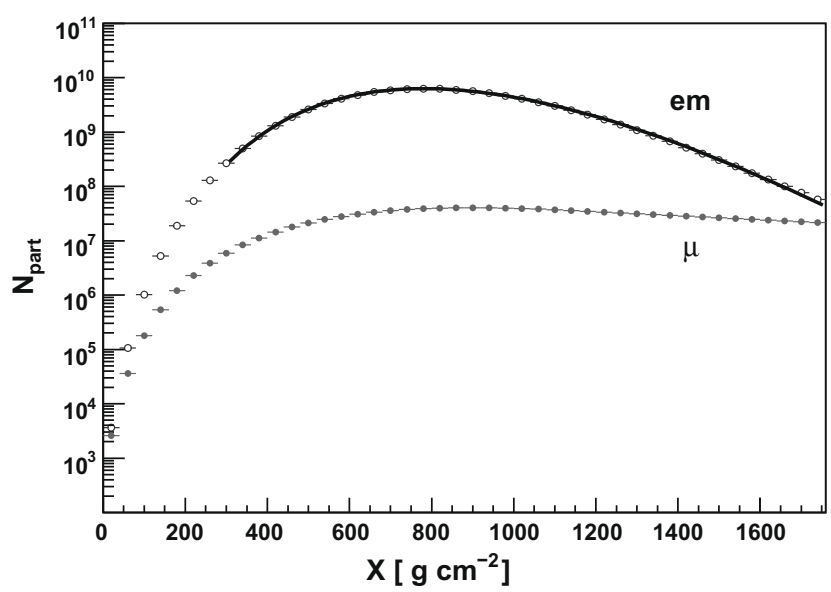

Fig. 1. Average longitudinal profile of three hundred proton-initiated showers with $E=10^{19} \mathrm{eV}$, and zenith angle $\theta=60^{\circ}$, simulated with CORSIKA-QGSJETII (open blue circles represent the electromagnetic component, red bullets the muonic one). The black continuous line is a fit of the electromagnetic profile with a Gaisser-Hillas function.

In the following, we first describe separately the effects on $S(1000)$ due to $P$, Section 2.1.1, and $\rho$, Section 2.1.2, and then in Section 2.1.3 we provide the full parameterisation of its variations as a function of changes in $P$ and $\rho$.

\subsubsection{Effect of air pressure variations on the SD signal}

From the point of view of $P$ (which measures the vertical air column density above ground), an increase (decrease) corresponds to an increased (decreased) matter overburden. This implies that the shower is older (younger), i.e. in a more (less) advanced stage when it reaches the ground level.

The longitudinal profile of the electromagnetic component of the EAS is exponentially attenuated beyond the shower maximum and can be described by a Gaisser-Hillas profile [7] (see Fig. 1). We are interested in the value of the electromagnetic signal measured at $1000 \mathrm{~m}$ from the core, referred hereafter as $S_{e m}$. The longitudinal development of the shower far from the core is delayed with respect to the one at the core, and can be parameterised as

$S_{e m}(E, X) \propto X^{\widehat{X}_{\max } / \Lambda} \exp \left[\left(\widehat{X}_{\max }-X\right) / \Lambda\right]$,

where $E$ is the primary energy, $X$ the slant depth, $\widehat{X}_{\max } \equiv X_{\max }+\Delta$ the average maximum of the shower at $1000 \mathrm{~m}$ from the core with $X_{\max }$ being the shower maximum, ${ }^{5} \Delta \simeq 150 \mathrm{~g} \mathrm{~cm}^{-2}$ is the typical increase of the shower maximum at $1000 \mathrm{~m}$ from the core [8] and $\Lambda \simeq 100 \mathrm{~g} \mathrm{~cm}^{-2}$ is the effective attenuation length after the maximum [9]. Therefore, a change in $P$ affects $S_{e m}$ :

$\frac{1}{S_{e m}} \frac{\mathrm{d} S_{e m}}{\mathrm{~d} P} \simeq-\frac{1}{g}\left[1-\frac{\widehat{X}_{\max }}{X}\right] \frac{\sec \theta}{\Lambda}$,

where $g \mathrm{~d} X=\mathrm{d} P \sec \theta$ is used, with $g$ the acceleration of gravity, and $\theta$ the shower zenith angle. Due to the flat longitudinal development of the muons (see Fig. 1), no significant pressure dependence is expected for the muonic component.

\subsubsection{Effect of air density variations on the SD signal}

Regarding $\rho$, this affects the Molière radius $r_{M}$

$r_{M} \equiv \frac{E_{s}}{E_{c}} \frac{X_{0}}{\rho} \simeq \frac{91 \mathrm{~m}}{\rho /\left(\mathrm{kg} \mathrm{m}^{-3}\right)}$,

\footnotetext{
${ }^{5} X_{\max } \simeq 750 \mathrm{~g} \mathrm{~cm}^{-2}$ for $10^{19} \mathrm{eV}$ showers according to the elongation rate measurement with the FD at the Pierre Auger Observatory [10].
}

where $E_{s} \equiv m_{e} c^{2} \sqrt{4 \pi / \alpha} \simeq 21 \mathrm{MeV}$ is the energy constant characterising the energy loss due to multiple Coulomb scattering, $E_{c} \simeq 86 \mathrm{MeV}$ is the critical energy in air and $X_{0} \simeq 37.1 \mathrm{~g} \mathrm{~cm}^{-2}$ is the radiation length in air. A variation in $r_{M}$ affects the lateral distribution of the electromagnetic component of the EAS, which can be approximately described with a Nishimura-Kamata-Greisen (NKG) profile $[11,12]$. At a large distance $r$ from the core, it behaves as $S_{e m}(r) \propto N_{e m}(r) \propto r_{M}^{-2}\left(r / r_{M}\right)^{-\eta}$, where $\eta \simeq 6.5-2 s$ and $s=3 X /(X+$ $\left.2 X_{\max }\right)$ is the age of the shower. Hence, a change in $\rho$ affects $S_{e m}$ :

$\frac{1}{S_{e m}} \frac{\mathrm{d} S_{e m}}{\mathrm{~d} \rho} \simeq \frac{(2-\eta)}{\rho}$.

In fact, the relevant value of $r_{M}$ is the one corresponding to the air density $\rho^{*}$ two radiation lengths above ground [12] in the direction of the incoming shower. This corresponds to $\simeq 700 \mathrm{~m} \cos \theta$ above the site of the Pierre Auger Observatory. On time scales of one day or more, the temperature gradient $(\mathrm{d} T / \mathrm{d} h)$ in the lowest layers of the atmosphere (the planetary boundary layer, which extends up to about $1 \mathrm{~km}$ above ground level) can be described by an average value of $\simeq-5.5^{\circ} \mathrm{C} \mathrm{km}^{-1}$ at the site of the Auger Observatory. Therefore, the variation of $\rho^{*}$ on temporal scales of one day essentially follows that of $\rho$. An additional effect is related to the diurnal variations of $\mathrm{d} T / \mathrm{d} h$, because during the day the surface of the Earth is heated by solar radiation, producing a steeper $\mathrm{d} T / \mathrm{d} h$ in the boundary layer. On the other hand, during the night the surface is cooled by the emission of long wavelength radiation: $\mathrm{d} T / \mathrm{d} h$ becomes smaller and even $T$ inversions can be observed before sunrise. As a result, the amplitude of the diurnal variation in $T$ (and $\rho$ ) is smaller at two radiation lengths above ground than at ground level. It is then useful to separate the daily modulation from the longer term one introducing the average daily density $\rho_{d}$ and the instantaneous departure from it, $\rho-\rho_{d}$. Therefore, the dependence of $S_{e m}$ on $\rho$ can be modeled by

$S_{e m}=S_{e m}^{0}\left[1+\alpha_{\rho}^{e m}\left(\rho_{d}-\rho_{0}\right)+\beta_{\rho}^{e m}\left(\rho-\rho_{d}\right)\right]$,

where $\rho_{0}=1.06 \mathrm{~kg} \mathrm{~m}^{-3}$ is chosen as the reference value of $\rho$ and is the average value measured at the site of the Pierre Auger Observatory over more than 3 years (1 January 2005 to 31 August 2008).

Concerning the muonic component of the signal at $1000 \mathrm{~m}$ from the core, $S_{\mu}$, its dependence on $\rho$ can be parameterised as

$S_{\mu}=S_{\mu}^{0}\left[1+\alpha_{\rho}^{\mu}\left(\rho_{d}-\rho_{0}\right)\right]$.

The $\rho$ dependence is written in terms of $\rho_{d}-\rho_{0}$ only because, as the muons are produced high in the atmosphere, their contribution to signal is not expected to depend on the daily modulations taking place in the boundary layer.

\subsubsection{Model of atmospheric effects on $S(1000)$}

The dependence of the total signal at $1000 \mathrm{~m}$ from the core, $S(1000) \equiv S=S_{e m}+S_{\mu}$, upon $P$ and $\rho$ can hence be written as

$S=S_{0}\left[1+\alpha_{P}\left(P-P_{0}\right)+\alpha_{\rho}\left(\rho_{d}-\rho_{0}\right)+\beta_{\rho}\left(\rho-\rho_{d}\right)\right]$

where $P_{0}=862 \mathrm{hPa}$ is the reference $P$ at the site of the Pierre Auger Observatory, $S_{0}$ is the value of the total signal at reference pressure and density $\left(P=P_{0}\right.$ and $\left.\rho=\rho_{d}=\rho_{0}\right)$, and

$\alpha_{P}=F_{e m} \alpha_{P}^{e m}, \quad \alpha_{\rho}=F_{e m} \alpha_{\rho}^{e m}+\left(1-F_{e m}\right) \alpha_{\rho}^{\mu}, \quad \beta_{\rho}=F_{e m} \beta_{\rho}^{e m}$,

where $F_{e m} \equiv S_{e m} / S$ is the electromagnetic fraction of the signal at $1000 \mathrm{~m}$ from the core. The values of $F_{e m}$ are obtained by means of proton-initiated showers simulated with CORSIKA-QGSJETII (see 


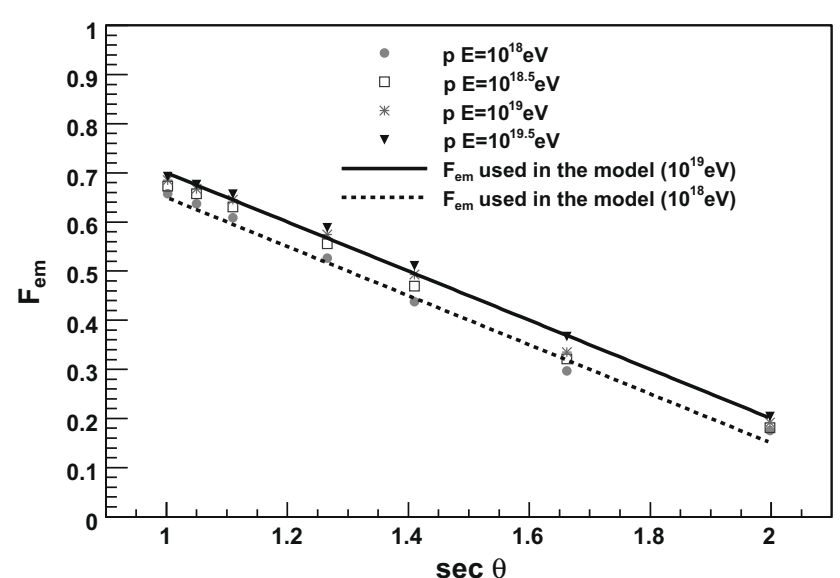

Fig. 2. Fraction of the total signal induced by the electromagnetic component of the shower at ground level at a distance of $1000 \mathrm{~m}$ from the shower axis $\left(F_{e m}\right)$ as a function of $\sec \theta$. A linear dependence of $F_{e m}$ on $\sec \theta$ (solid and dashed lines) is assumed in this work.

Section 4): they decrease approximately linearly with $\sec \theta$ for all the simulated primary energies (see Fig. 2).

We will adopt hereafter

$F_{e m}=F_{e m}^{v}-0.5(\sec \theta-1)$,

where $F_{e m}^{v} \equiv F_{e m}(\theta=0)$ varies between $\approx 0.65$ at $10^{18} \mathrm{eV}$ and $\approx 0.7$ at $10^{19} \mathrm{eV}$. We note that since the inferred electromagnetic fraction depends on the hadronic model adopted and on the CR composition assumed, the actual value of $F_{e m}$ may be different. As shown in [9], for iron-induced showers the simulated $S_{\mu}$ is $40 \%$ higher than in the case of protons, while the SIBYLL model [13] predicts a muonic signal 13\% lower than QGSJETII for both proton and iron primaries. The corresponding variation $F_{e m}^{v}$ at a primary energy of $10^{19} \mathrm{eV}$ would be $\simeq-11 \%$ for iron with respect to proton, and $\simeq+4 \%$ for SIBYLL simulations with respect to QGSJETII.

Finally, with respect to the coefficients in Eq. (4):

(i) For the pressure coefficient, we have from Eq. (1)

$\alpha_{P}^{e m} \simeq-\frac{1}{g}\left[1-\frac{\widehat{X}_{\max }}{X}\right] \frac{\sec \theta}{\Lambda}$,

where $X=X_{v} \sec \theta$ and $X_{v} \simeq 880 \mathrm{~g} \mathrm{~cm}^{-2}$ is the atmospheric depth at the site of the Pierre Auger Observatory.

(ii) From Eq. (2)

$\alpha_{\rho}^{e m} \simeq-\frac{4.5-2 s}{\rho}$,

where $s=3 /\left(1+2 \cos \theta X_{\max } / X_{v}\right)$, with $X_{\max } / X_{v} \simeq 0.85$ for $10^{19} \mathrm{eV}$ primaries. Pressure effects associated to the change in the slope of the lateral distribution function due to the $X$ dependence of $s$ are negligible.

(iii) The coefficient $\beta_{\rho}^{e m}$ should be smaller than $\alpha_{\rho}^{e m}$ (in absolute value) reflecting the reduction in the amplitude of the $\rho-\rho_{d}$ variations two radiation lengths above ground level. The difference should also depend on $\theta$. For instance, assuming an exponential decrease of the density amplitude with the height $h$

$\rho(h)-\rho_{d}(h)=\exp \left(-a \frac{h}{700 \mathrm{~m}}\right)\left[\rho(0)-\rho_{d}(0)\right]$

would lead to

$\beta_{\rho}^{e m} \simeq \exp (-a \cos \theta) \alpha_{\rho}^{e m}$, where $a$ parameterises the amplitude of the daily density variation in the lower atmosphere and is completely independent of the shower development. It characterises the scale height for the decrease of the daily thermal amplitude, which becomes $1 / e$ of its ground value at a height $(700 \mathrm{~m}) / a$. The value of $a$ is expected to be of order unity.

(iv) The coefficient $\alpha_{\rho}^{\mu}$ is expected to be small, and will be assumed to be independent of $\theta$, because of the relatively flat longitudinal development of the muons as shown in Fig. 1. Its value will be taken to be zero since the air shower simulations are consistent with a vanishing $\alpha_{\rho}^{\mu}$ coefficient (see Section 4).

\subsection{Atmospheric effects on the event rate}

The dependence of the measured signal on variations of $P$ and $\rho$ produces also a modulation of the rate of recorded events. The trigger probability, $P_{t r}$, is a well-defined function of the signal [4]. As atmospheric variations correspond to signal variations, this implies that the same primary particle (in particular, with the same primary energy) will induce different signals depending on $P$ and $\rho$. This in turn affects the probability for the shower to trigger the SD array.

The effect can be quantified starting from the relation between $S(1000)$ and the energy of the primary cosmic ray. In the case of the Pierre Auger Observatory, the primary energy is reconstructed as

$E_{r} \propto[S(1000)]^{B}$,

where $B=1.08 \pm 0.01$ (stat) \pm 0.04 (sys) is derived from the calibration of the SD energy using the FD energy measurement [14]. Following Eq. (3), the primary energy $E_{0}(\theta, P, \rho)$ that would have been obtained for the same shower at the reference pressure $P_{0}$ and density $\rho_{0}$, is related to $E_{r}$ as follows:

$E_{0}=E_{r}\left[1-\alpha_{P}\left(P-P_{0}\right)-\alpha_{\rho}\left(\rho_{d}-\rho_{0}\right)-\beta_{\rho}\left(\rho-\rho_{d}\right)\right]^{B}$.

In a zenith angle bin $\mathrm{d} \theta$, the rate $R$ of events per unit time and unit solid angle above a given signal $S_{\min }$ can be written as

$\frac{\mathrm{d} R}{\mathrm{~d} \theta}\left(\theta, S_{\min }\right)=\frac{\mathrm{d} A}{\mathrm{~d} \theta}(\theta) \int_{S_{\min }} \mathrm{d} S P_{t r}(S) \frac{\mathrm{d} J}{\mathrm{~d} S}$,

where $A$ is the geometrical aperture and $J$ is the flux of cosmic rays.

Assuming that the cosmic ray spectrum is a pure power law, i.e. $\mathrm{d} J / \mathrm{d} E_{0} \propto E_{0}^{-\gamma}$, using Eq. (7), and neglecting the small energy dependence of the weather coefficients, we find that

$$
\begin{aligned}
\frac{\mathrm{d} J}{\mathrm{~d} S} \propto & E_{0}^{-\gamma} \frac{\mathrm{d} E_{0}}{\mathrm{~d} S} \propto S^{-B \gamma+B-1}\left[1+B(\gamma-1)\left(\alpha_{P}\left(P-P_{0}\right)\right.\right. \\
& \left.\left.+\alpha_{\rho}\left(\rho_{d}-\rho_{0}\right)+\beta_{\rho}\left(\rho-\rho_{d}\right)\right)\right] .
\end{aligned}
$$

From the dependence on the atmosphere of the measured CR flux above a given signal, we derive the corresponding dependence of the rate of events. If $S_{\min }$ is the minimum required signal at $1000 \mathrm{~m}$ from the core to trigger the array

$$
\begin{aligned}
& \frac{\mathrm{d} R}{\mathrm{~d} \theta} \propto {\left[1+a_{P}\left(P-P_{0}\right)+a_{\rho}\left(\rho_{d}-\rho_{0}\right)+b_{\rho}\left(\rho-\rho_{d}\right)\right] } \\
& \times \int_{S_{\min }} \mathrm{d} S P_{t r}(S) S^{-B \gamma+B-1}
\end{aligned}
$$

with the integral on the right-hand side being independent of the weather variations. The coefficients $a_{P}, a_{\rho}$ and $b_{\rho}$ are then related to the coefficients describing the modulation of the signal by $a_{\rho, P}=B(\gamma-1) \alpha_{\rho, P}$ and $b_{\rho}=B(\gamma-1) \beta_{\rho}$. 

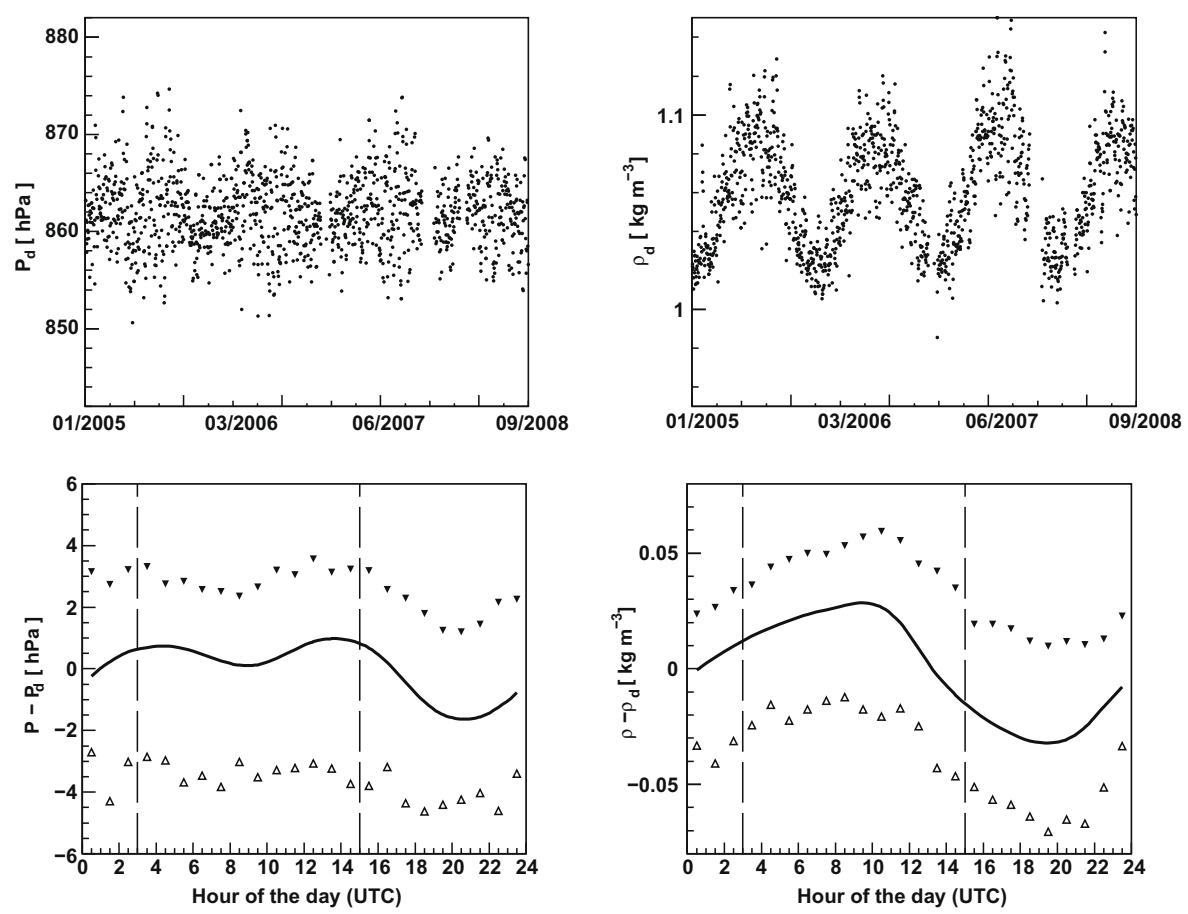

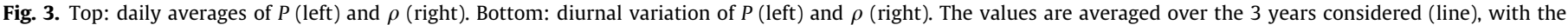
maximum and minimum variations marked by black and white triangles. The local time is UTC-3 h (vertical lines mark local midnight and noon).

\section{Atmospheric effects on the experimental rate of events}

To study the modulation of the rate of events, we use data taken by the SD from 1 January 2005 to 31 August 2008. All events with $\theta<60^{\circ}$ are used, for a total of about 960000 showers with a median energy $6 \times 10^{17} \mathrm{eV}$. These are selected on the basis of the topology and time compatibility of the triggered detectors [4]. The station with the highest signal must be enclosed within an active hexagon, in which all six surrounding detectors were operational at the time of the event.

At the site of the Pierre Auger Observatory, the ground temperature and pressure are measured every $5 \mathrm{~min}$. The air density is given by: $\rho=\left(M_{m} / R\right)(P / T)$ where $M_{m}$ is the molecular mass of air and $R$ is the gas constant. The daily average density $\rho_{d}$ is obtained with a smoothing procedure consisting in taking, for each time, the average value of $\rho$ over a 24-h interval centered at the time of interest. The daily and diurnal variations of the ground $P$ and $\rho$ are shown in Fig. 3 (upper and lower panels respectively).

The pressure exhibits less than $\pm 2 \%$ variation during the period considered, while $\rho_{d}$ changes up to a maximum of $\pm 8 \%$ with an additional diurnal variation of density which is of $\pm 3 \%$ on average with maximum values of ${ }_{-8}+6 \%$.

In the period under study, the number of surface detectors steadily increased from about 700 to about 1590 . To take this into account, rather than using the raw number of triggering events, we compute the rate every hour normalised to the sensitive area, which is calculated every second from the total area of the active hexagons. The daily and the diurnal rate of events are presented in Fig. 4 (black points), where it is evident that they both follow qualitatively the corresponding modulations of pressure and density from Fig. 3.

We use the expression given by Eq. (8) to fit the measured rate of events. Assuming that the number of events $n_{i}$ observed in each hour bin $i$ follows a Poisson distribution of average $\mu_{i}$, a maximum likelihood fit is performed to estimate the coefficients $a_{P}, a_{\rho}$ and $b_{\rho}$.
The likelihood function is $L=\prod \frac{\mu_{i}^{n_{i}}}{n_{i} !} e^{-\mu_{i}}$. The expected number of events in bin $i$ is given by

$\mu_{i}=R_{0} \times A_{i} \times C_{i}$,

where $R_{0}$ is the average rate we would have observed if the atmospheric parameters were always the reference ones, i.e. $R_{0}=\frac{\sum n_{i}}{\sum A_{i} C_{i}}$, with $A_{i}$ the sensitive area in the $i$ th bin and, according to Eq. (8), $C_{i}$ is

$C_{i}=\left[1+a_{P}\left(P_{i}-P_{0}\right)+a_{\rho}\left(\rho_{d_{i}}-\rho_{0}\right)+b_{\rho}\left(\rho_{i}-\rho_{d_{i}}\right)\right]$.

The fitted parameters are:

$a_{P}=(-0.0027 \pm 0.0003) \mathrm{hPa}^{-1}$,

$a_{\rho}=(-1.99 \pm 0.04) \mathrm{kg}^{-1} \mathrm{~m}^{3}$,

$b_{\rho}=(-0.53 \pm 0.05) \mathrm{kg}^{-1} \mathrm{~m}^{3}$,

corresponding to a reduced $\chi^{2}$ of 1.06 , where $\chi^{2}=\sum_{i}\left(n_{i}-\mu_{i}\right)^{2} / \mu_{i}$. The result of the fit is shown in Fig. 4, compared to the daily-averaged and the shorter term modulations of the measured event rate.

To check the stability of the coefficients with respect to the energy, the same study has been done for the subset of events with a reconstructed energy above $10^{18} \mathrm{eV}$, corresponding to $\simeq 20 \%$ of the total statistics. The fitted coefficients are consistent within the fit uncertainties. A more detailed study of the energy dependence of the coefficients will become feasible in future with increased statistics.

\section{Atmospheric effects on simulated air showers}

To complete the study of atmospheric effects, we performed full EAS simulations in different atmospheric conditions. We simulated proton-initiated showers using the CORSIKA code [15] with hadronic interaction models QGSJETII [16] and Fluka [17].

We considered four fixed energies of the primary particle $\left(E=10^{18} \mathrm{eV}, 10^{18.5} \mathrm{eV}, 10^{19} \mathrm{eV}\right.$ and $10^{19.5} \mathrm{eV}$ ) and seven fixed zenith 

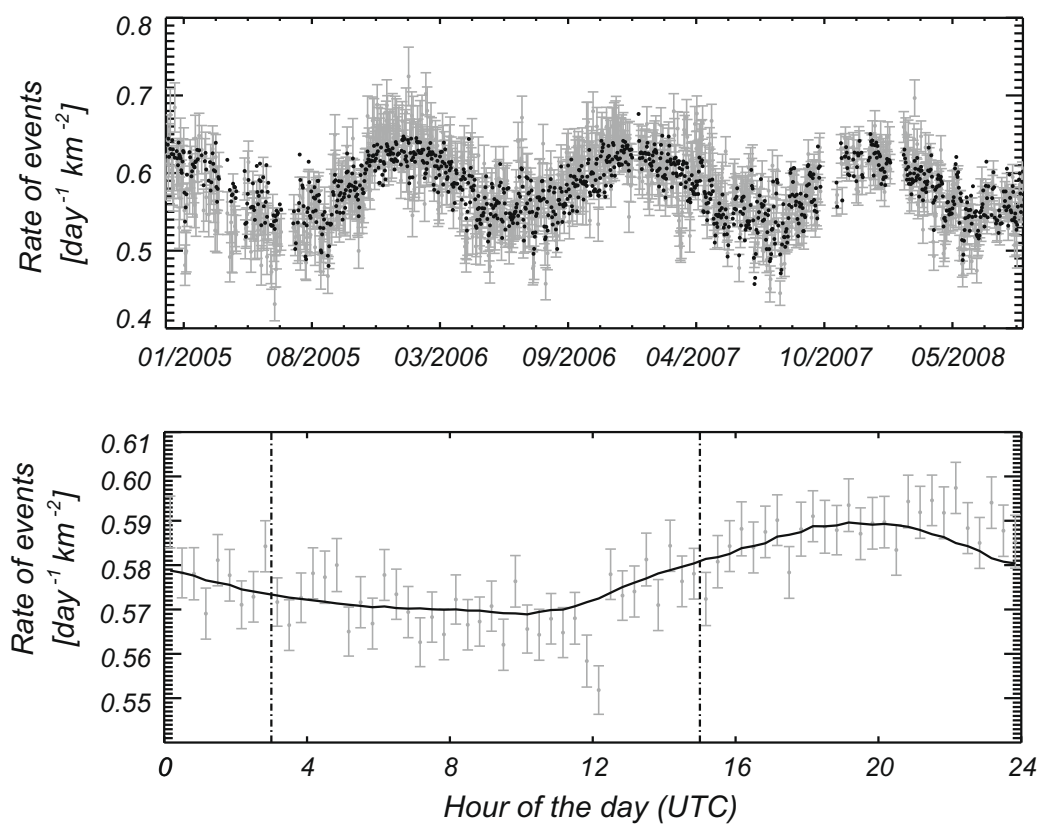

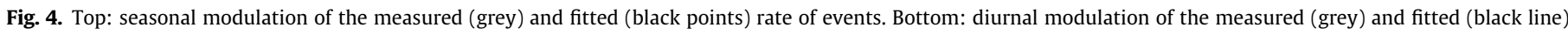
event rate.
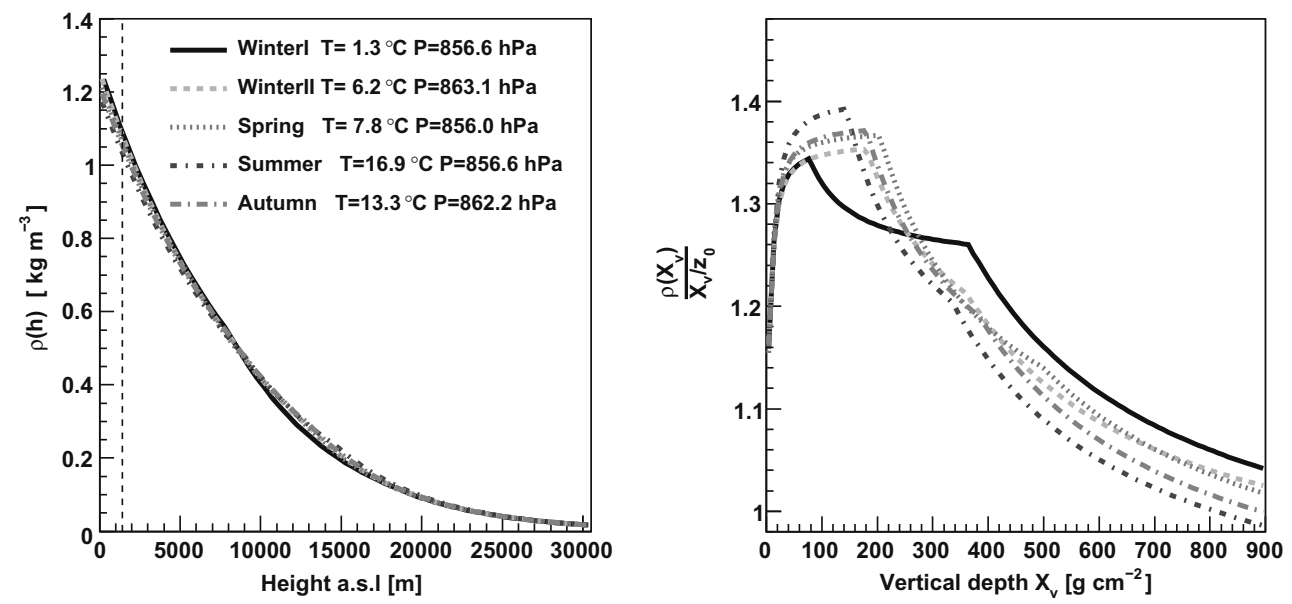

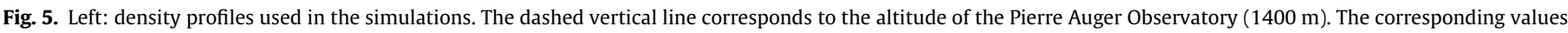
of ground $P$ and $T$ are given in the legend. Right: same density profiles normalised to an isothermal one $\left(\rho\left(X_{v}\right)=X_{v} / z_{0}\right.$ with $\left.z_{0}=8.4 \mathrm{~km}\right)$.

angles between $\theta=0^{\circ}$ and $\theta=60^{\circ}$. For the air density profiles, we used five parameterisations (shown in Fig. 5) of the seasonal average of radio sounding campaigns carried out at the site of the Pierre Auger Observatory [6] over a wide range of variation in temperature. $^{6}$ The set of simulations consists of 60 showers for each combination of atmospheric profile, energy and angle with an optimal statistical thinning level of $10^{-6}[18,19]$.

To compare with model predictions and data, we need to determine for each combination $(E, \theta)$ the dependence of $S(1000)$ on the variations of $P$ and $\rho$. The signal can be estimated through simpli-

\footnotetext{
${ }^{6}$ The atmospheric profiles are implemented in the CORSIKA code through the dependence of $X$ on $h . P, \rho$ and $T$ profiles can be derived from: $\rho(h)=-\mathrm{d} X / \mathrm{d} h$ and $P(h)=g X(h)$. The ground values in Fig. 5 are computed at an observation level $h=1400 \mathrm{~m}\left(\simeq 880 \mathrm{~g} \mathrm{~cm}^{-2}\right)$, corresponding to the altitude of the Pierre Auger Observatory.
}

fied assumptions about the energy deposited by particles on the basis of their kinetic energy $E_{k}$ :

(i) $\mathrm{e}^{-} \mathrm{e}^{+}$deposit $E_{k}-\epsilon_{t h}$, where $\epsilon_{t h}=260 \mathrm{keV}$ is the energy threshold for Cherenkov emission in water.

(ii) photons deposit $E_{k}-2 m_{e}-2 \epsilon_{t h}$.

(iii) muons deposit $240 \mathrm{MeV}$ corresponding to the average energy released by a vertical muon crossing a $1.2 \mathrm{~m}$ high water-Cherenkov tank.

The contribution of each particle is multiplied by the weight assigned by the thinning algorithm. We obtain the Cherenkov signal per unit area perpendicular to the shower plane $C_{s p}(r)$. For the muons, the Cherenkov signal is proportional to the track length in the station so that: $C^{\mu}=C_{s p}^{\mu}$, whereas for the electromagnetic component: $C^{e m}=\cos \theta C_{s p}^{e m}$. 
The left panel of Fig. 6 shows the lateral distribution $C(r)=C^{e m}(r)+C^{\mu}(r)$, which is proportional to $S(1000)$, for four atmospheres (relative to the Spring one) in the case of $E=10^{19} \mathrm{eV}$ and $\theta=18^{\circ}$. The effect related to the Molière radius can be clearly seen as a broadening of the lateral distribution with increasing temperature.



To derive the atmospheric coefficients, we correlate the simulated $C(1000)$ (taken as the average signal between $950 \mathrm{~m}$ and $1050 \mathrm{~m}$ ) with $P$ and $\rho$ (see Eq. (3)). Since we are using seasonal atmospheric profiles, we do not have access to the diurnal variation of $T$ and thus we cannot determine the coefficient $\beta_{\rho}$ related to the diurnal variation of $\rho$. The two coefficients $\alpha_{\rho}$ and $\alpha_{P}$ can

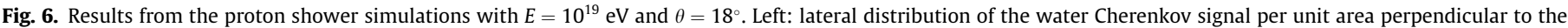

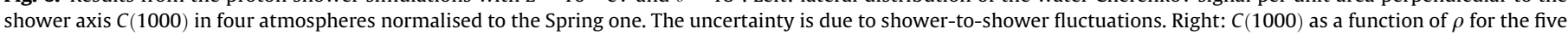
atmospheres considered. The dashed and the continuous lines are the projections of the fit in the $(C(1000), \rho)$ plane for $P=856$ hPa and $P=862$ hPa, respectively.
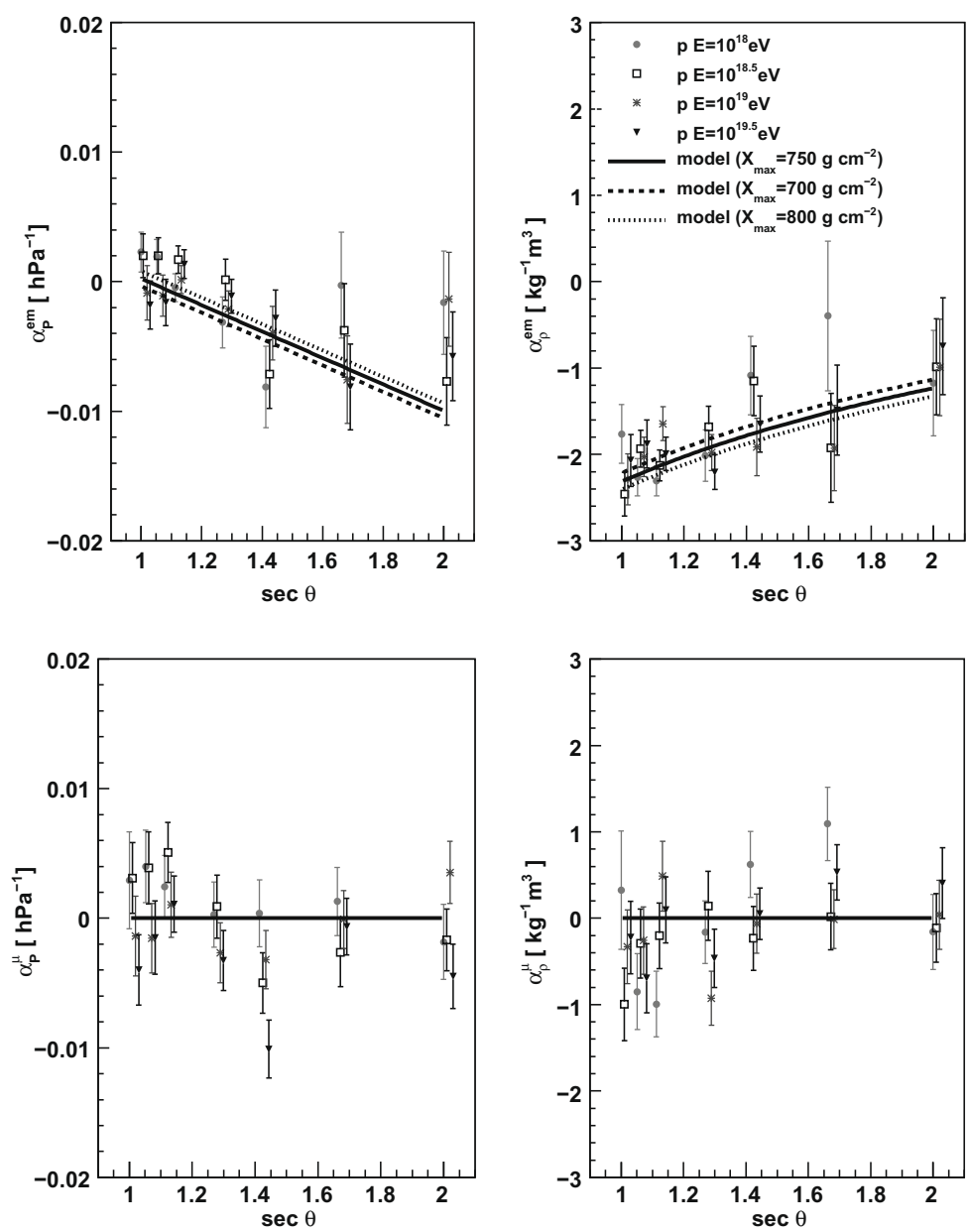

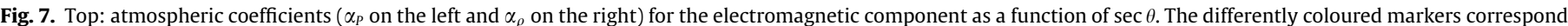

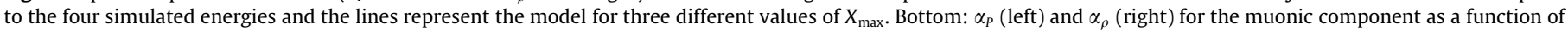
$\sec \theta$. 
be determined for each fixed energy and angle with a two dimensional fit of the $C(1000)$, obtained for the five atmospheric profiles, as function of $\rho$ and $P$. As an example, we show in Fig. 6 (right) the results of the fit for the case of $E=10^{19} \mathrm{eV}$ and $\theta=18^{\circ}$, projected on the $(C(1000), \rho)$ plane for the sake of clarity. Moreover, in the case of simulations we are able to separate the electromagnetic and the muonic contribution to the signal and thus to determine the atmospheric coefficients for each component (see Fig. 7).

\section{Comparison among model, data and simulations}

In this section, we compare the atmospheric coefficients derived from data with those expected from the model and simulations. We recall that with the simulations we cannot access the coefficient $\beta_{\rho}$, as we use average seasonal profiles for the atmosphere, while we can investigate the behaviour of separate coefficients for the electromagnetic and muonic components of EAS. On the other hand, with experimental data we cannot separate the electromagnetic and muonic components, while we can fully investigate the diurnal effects of atmospheric changes and compare measurements and expectations for all of the three coefficients.

The comparison between atmospheric coefficients for the electromagnetic and muonic components of EAS from simulations and model is shown in Fig. 7, as a function of $\sec \theta$. With respect to the electromagnetic part, the model predictions for both the $P$ and $\rho$ coefficients, and their dependence on the shower zenith angle, are reasonable at all energies. Concerning the muonic component
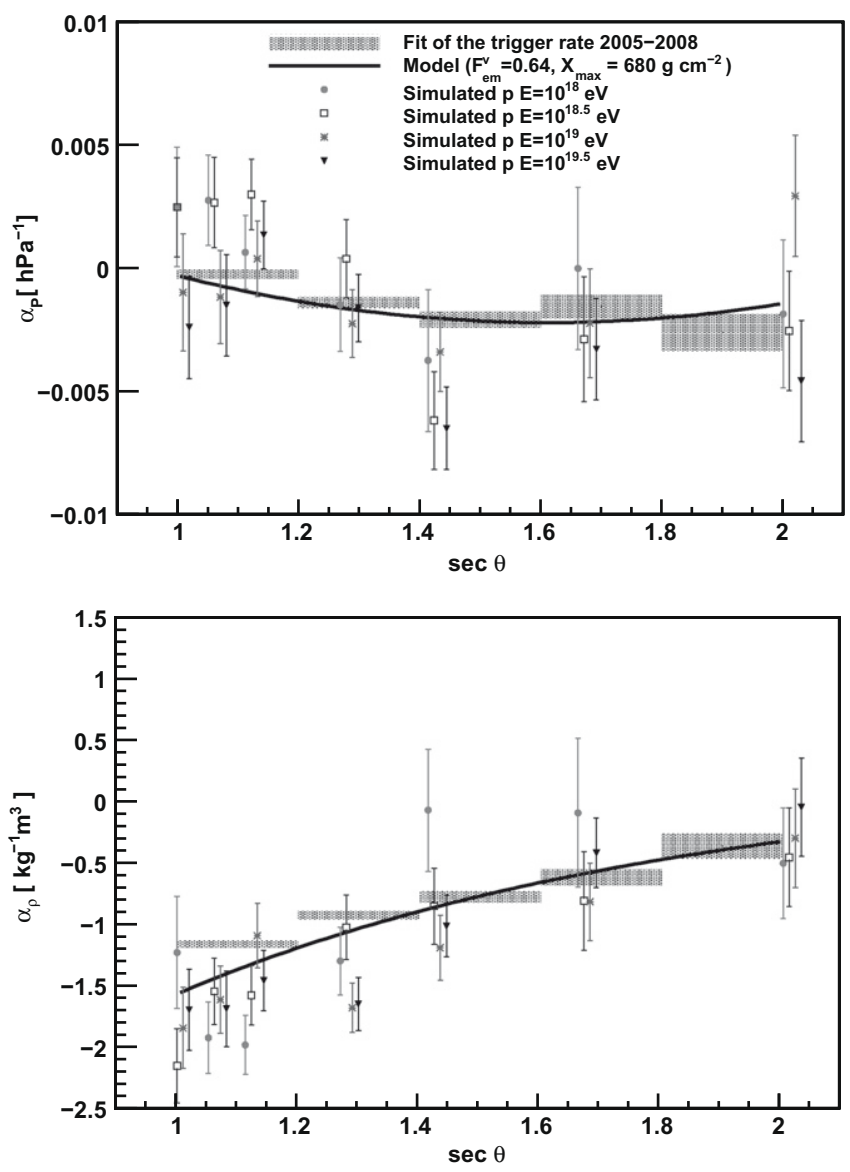

Fig. 8. Comparison of the $P$ coefficients (top) and of the daily density coefficients (bottom) as a function of $\sec \theta$ obtained from data (grey shaded rectangle), simulations (bullets) and model (continuous line).

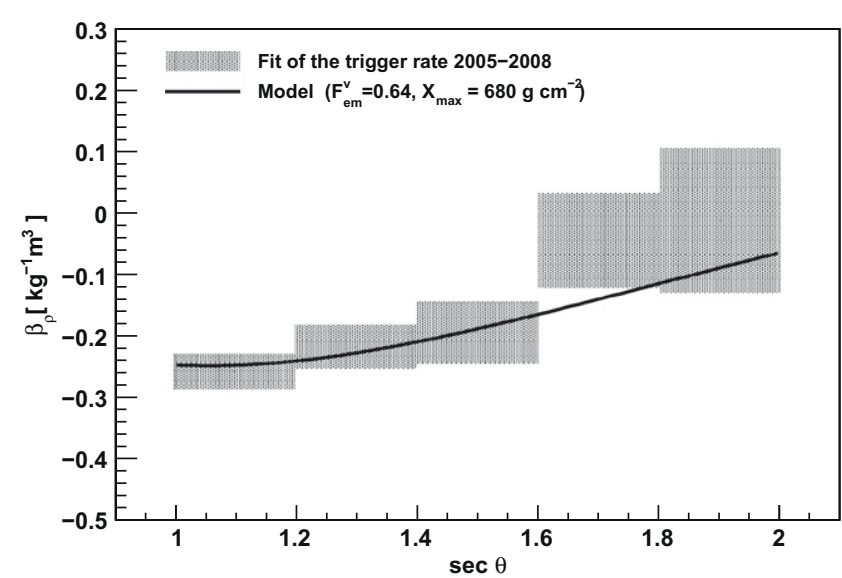

Fig. 9. Comparison of $\beta_{\rho}$ from data with model. A fit to the data points is performed to get the value of the parameter $a=1.7 \pm 0.1$ (see Eq. (6)).

of the signal and its dependence on $P, \alpha_{P}^{\mu}$ is compatible with zero at all energies, as expected from the flat longitudinal development of the number of muons. For the dependence on $\rho$, the model is not predictive but from the simulations we get a value of $\alpha_{\rho}^{\mu}$ compatible with zero. This justifies the adoption in the model of vanishing coefficients for the muonic component.

The comparison of the global coefficients as a function of $\sec \theta$ is done for $\alpha_{P}, \alpha_{\rho}$ and $\beta_{\rho}$ in Figs. 8 and 9. In the case of the data, the dependence on $\theta$ has been studied by dividing the data set in subsets corresponding to five bins of equal width in sec $\theta$. For each subset the same fitting procedure as illustrated in Section 3 is used. The signal coefficients are then derived by dividing the rate coefficients by $B(\gamma-1)$ (see the end of Section 2.2). Since the bulk of the triggering events have an energy $<10^{18} \mathrm{eV}$, we used $\gamma=3.30 \pm 0.06$, as measured with the Auger Observatory below $10^{18.65} \mathrm{eV}$ [20].

The comparison among data, simulations and model is shown for the pressure coefficient $\alpha_{P}$ and the daily component of the density coefficient $\alpha_{\rho}$ in Fig. 8 (top and bottom, respectively). In the model, we use the value of $X_{\max }$ measured by the Auger Observatory at the median energy of the triggering events [10], and a $F_{e m}^{v}$, corresponding at the same energy, obtained under the assumption that $F_{e m}^{v}$ scales linearly with the logarithm of the primary energy. The reduced $\chi^{2}$ for the data-model comparison is 3.3 for $\alpha_{P}$ and 11.0 for $\alpha_{\rho}$. For the instantaneous density coefficient $\beta_{\rho}$, the comparison between data and model is shown in Fig. 9. The data-model comparison gives in this case a reduced $\chi^{2}$ of 0.6 .

\section{Conclusions}

We have studied the effect of atmospheric variations (in $P, T$ and $\rho$ ) on extensive air showers using about 960000 events collected by the surface detector of the Pierre Auger Observatory from 1 January 2005 to 31 August 2008. We observe a significant modulation of the rate of events with the atmospheric variables, both on a seasonal scale $(\sim 10 \%)$ and on a shorter time scale $(\sim 2 \%$ on average during a day). This modulation can be explained as due to the impact of the density and pressure changes on the shower development, which affects the energy estimator $S(1000)$, the size of the shower signal $1000 \mathrm{~m}$ from the shower axis. This affects the trigger probability and the rate of events above a fixed energy.

The dominant effect is due to the change with the air density of the Molière radius near ground. It induces a variation of the rate of events with associated correlation coefficients of $(-1.99 \pm$ $0.04) \mathrm{kg}^{-1} \mathrm{~m}^{3}$ and $(-0.53 \pm 0.05) \mathrm{kg}^{-1} \mathrm{~m}^{3}$ on long and short time scales, respectively. 
The second effect is due to the pressure changes, which affect, through the variation of the amount of matter traversed, the stage of development of the showers when they reach ground. The impact of the pressure variation on the rate amounts to $(-2.7 \pm$ $0.3) \times 10^{-3} \mathrm{hPa}^{-1}$.

Comparing the coefficients obtained from data, shower simulations in different atmospheric profiles and expectations from the model built, a good agreement is obtained, not only for the overall size of the effect but also for the zenith angle dependence.

Taking into account the atmospheric effects will allow to reduce the systematics in the energy reconstruction. Furthermore, it will be possible to correct for the seasonal modulation, which can affect the search for large scale anisotropies.

\section{Acknowledgements}

The successful installation and commissioning of the Pierre Auger Observatory would not have been possible without the strong commitment and effort from the technical and administrative staff in Malargüe.

We are very grateful to the following agencies and organizations for financial support: Comisión Nacional de Energía Atómica, Fundación Antorchas, Gobierno De La Provincia de Mendoza, Municipalidad de Malargüe, NDM Holdings and Valle Las Leñas, in gratitude for their continuing cooperation over land access, Argentina; the Australian Research Council; Conselho Nacional de Desenvolvimento Científico e Tecnológico (CNPq), Financiadora de Estudos e Projetos (FINEP), Fundação de Amparo à Pesquisa do Estado de Rio de Janeiro (FAPERJ), Fundação de Amparo à Pesquisa do Estado de São Paulo (FAPESP), Ministério de Ciência e Tecnologia (MCT), Brazil; AVCR AV0Z10100502 and AV0Z10100522, GAAV KJB300100801 and KJB100100904, MSMT-CR LA08016, LC527, 1M06002, and MSM0021620859, Czech Republic; Centre de Calcul IN2P3/CNRS, Centre National de la Recherche Scientifique (CNRS), Conseil Régional Ile-de-France, Département Physique Nucléaire et Corpusculaire (PNC-IN2P3/CNRS), Département Sciences de l'Univers (SDU-INSU/CNRS), France; Bundesministerium für Bildung und Forschung (BMBF), Deutsche Forschungsgemeinschaft (DFG), Helmholtz-Gemeinschaft Deutscher Forschungszentren (HGF), Finanzministerium Baden-Württemberg, Ministerium für Wissenschaft und Forschung, Nordrhein-Westfalen, Ministerium für Wissenschaft, Forschung und Kunst, Baden-Württemberg, Germany; Istituto Nazionale di Fisica Nucleare (INFN), Ministero dell'Istruzione, dell'Università e della Ricerca (MIUR), Italy; Consejo Nacional de Ciencia y Tecnología (CONACYT), Mexico; Ministerie van Onderwijs, Cultuur en Wetenschap, Nederlandse Organisatie voor Wetenschappelijk Onderzoek (NWO), Stichting voor Fundamenteel Onderzoek der Materie (FOM), The Netherlands; Ministry of Science and Higher Education, Grant Nos. 1 P03 D 014 30, N202 090 31/0623, and PAP/218/2006, Poland; Fundação para a Ciência e a Tecnologia, Portugal; Ministry for Higher Education, Science, and Technology, Slovenian Research Agency, Slovenia; Comunidad de Madrid, Consejería de Educación de la Comunidad de Castilla La Mancha, FEDER funds, Ministerio de Ciencia e Innovación, Xunta de Galicia, Spain; Science and Technology Facilities Council, United Kingdom; Department of Energy, Contract No. DE-AC0207CH11359, National Science Foundation, Grant No. 0450696, The Grainger Foundation USA; ALFA-EC/ HELEN, European Union 6th Framework Program, Grant No. MEIF-CT-2005-025057, European Union 7th Framework Program, Grant No. PIEF-GA-2008220240, and UNESCO.

\section{References}

[1] J. Abraham et al., [The Pierre Auger Collaboration], Nucl. Instrum. Meth. A 523 (2004) 50.

[2] I. Allekotte et al., Nucl. Instrum. Meth. A 586 (2008) 409.

[3] J. Abraham et al. [The Pierre Auger Collaboration], The fluorescence detector of the Pierre Auger Observator", in preparation, arxiv:0907.4282.

[4] D. Allard et al. [The Pierre Auger Collaboration], in: Proc. 29th Int. Cosmic Ray Conf., vol. 7, Pune, India, 2005, p. 287.

[5] D. Newton et al., Astropart. Phys. 26 (2007) 414.

[6] B. Keilhauer et al., Astropart. Phys. 22 (2004) 249; J. Blümer et al. [The Pierre Auger Collaboration], in: Proc. 29th Int. Cosmic Ray Conf., vol. 7, Pune, India, 2005, p. 123.;

B. Keilhauer et al., Astropart. Phys. 25 (2006) 259

[7] T.K. Gaisser, A.M. Hillas, in: Proc. 15th Int. Cosmic Ray Conf., vol. 8, Plovdiv, Bulgaria, 1977, p. 353.

[8] P. Billoir, C. Roucelle, J.C. Hamilton, astro-ph/0701583.

[9] F. Schmidt et al., Astropart. Phys. 29 (2008) 355.

[10] M. Unger [The Pierre Auger Collaboration], in: Proc. 30th Int. Cosmic Ray Conf., vol. 4, Merida, Mexico, 2007, p. 373.

[11] K. Kamata, J. Nishimura, Prog. Theor. Phys. 6 (1958) 93.

[12] K. Greisen, Prog. Cosmic Ray Phys. 3 (1956) 1.

[13] J. Engel et al., Phys. Rev. D46 (1992) 5013; R.S. Fletcher et al., Phys. Rev. D50 (1994) 5710; R. Engel et al., in: Proc. 26th Int. Cosmic Ray Conf., vol. 1, Salt Lake Cuty, USA, 1999, p. 415.

[14] J. Abraham et al., [The Pierre Auger Collaboration], Phys. Rev. Lett. 101 (2008) 061101.

[15] D. Heck et al., Report FZKA 6019, 1998.

[16] S.S. Ostapchenko, Nucl. Phys. B (Proc. Suppl.) 151 (2006). 143 and 147.

[17] A. Fassò et al., CERN-2005-10, INFN/TC_05/11, SLAC-R-773, 2005.; A. Fassò et al., CHEP2003, La Jolla, USA (paper MOMT005), arXiv:hep-ph/ 0306267

[18] A.M. Hillas, Nucl. Phys. B (Proc. Suppl.) 52 (1997) 29.

[19] M. Kobal, [The Pierre Auger Collaboration], Astropart. Phys. 15 (2001) 259.

[20] T. Yamamoto [The Pierre Auger Collaboration], in: Proc. 30th Int. Cosmic Ray Conf., vol. 4, Merida, Mexico, 2007, p. 335. 